\title{
Ant-Based Mission Planning: Two Examples
}

\author{
Evridiki V. Ntagiou* and Roberto Armellin ${ }^{\dagger}$ \\ Surrey Space Centre, University of Surrey, Guildford, GU2 7XH, United Kingdom \\ Claudio Iacopino $¥$ \\ Surrey Satellite Technology, Guildford, GU2 7YE, United Kingdom \\ Nicola Policella ${ }^{\S}$ and Alessandro Donati $₫$ \\ ESOC, European Space Agency, Robert-Bosch-Strasse 5, 64293 Darmstadt, Germany
}

\begin{abstract}
The Earth Observation market is growing rapidly, along with the missions' complexity. Therefore, automated Mission Planning systems are being designed, allowing for operators to simply specify their intentions on a high level. In this paper, we propose an automated Mission Planning System based on the ants' foraging mechanism and apply it to two different mission planning problems, from an Earth Imaging and a Data Relay mission, investigating the system's ability to be generalised. We compare the planning process for the two problems and generalise on the type of planning problems the system can address.
\end{abstract}

\section{Introduction}

Earth Observation (EO) market has been in the spotlight in the last decades due to a growing demand from users for the data that EO satellites provide us with, ranging from weather forecast and science applications to natural disaster data. As a result, an increasing number of EO missions are being launched, usually consisting of multiple spacecraft. EO missions are usually equipped with complex instruments of many capabilities, a fact that leads to the exponential increase of the spacecraft's possible states at each moment. Together with the more complicated objectives of the missions, the process of Planning and scheduling $(\mathrm{P} \& \mathrm{~S})$ has become too complex for human operators to handle. Automated $\mathrm{P} \& \mathrm{~S}$ systems are designed and implemented, producing either feasible mission schedules ${ }^{1,2]}$ or optimal ones ${ }^{3[4, \sqrt[4]{5}}$ in which the satellite resources' management is optimized based on the mission objective. In an automated system, the operator defines a goal on a higher level, and the system is responsible for defining the activities that will help achieving the goal. This is the concept of Goal Based operations, ${ }^{6}$ that simplifies the operator workload and offers flexibility in ordering activities.

A critical issue of the current way Mission Planning Systems (MPS) are designed, is that a small part of the software can be reused in different missions. The largest part of the MPS is designed for each new mission from scratch, ${ }^{7}$ which is costly in both time and budget. Ongoing research on designing a generalised and reusable MPS that will produce timelines for multiple diverse missions has been published $8,9,10$ and enjoys great acceptance from the community of Space Operations.

In this paper, we propose a MPS architecture based on the ants' foraging mechanism. We investigate the application of the approach on the planning problems that appear in 2 different missions: Surrey Satellite Technology Limited (SSTL) Disaster Monitoring Constellation (DMC3) and a Data Relay System. The planning problems of those two missions differ in their constraints and objectives but not in complexity, as they are both $N P$-hard, like many scheduling problems found in the space industry. The system's input consists of the mission capabilities, objectives, constraints and other mission specific information.

*PhD Student, Surrey Space Centre, e.ntagiou@surrey.ac.uk

${ }^{\dagger}$ University Lecturer, Surrey Space Centre, r.armellin@surrey.ac.uk

${ }_{\ddagger}^{\ddagger}$ Mission Planning Engineer, Ground Software Team, c.iacopino@sstl.co.uk

$\S$ Senior Research Engineer, Advanced Mission Concepts and Technologies, nicola.policella@esa.int

『Section Head, Advanced Mission Concepts and Technologies, alessandro.donati@esa.int 
The problem model is a weighted directed graph generated based on this information. The model is used as input in an Ant Colony Optimization (ACO) algorithm that solves the planning problem, producing feasible timelines. The rest of the paper is divided as follows: in Section II we describe the two missions and formally define the planning problems. In Section III we present the main components of the MPS architecture: problem representation and planning algorithm. After contrasting the planning process for the two problems, we discuss the potential of the proposed MPS within a generalized Mission Planning framework and briefly present our future work in Section IV]

\section{The two Case Studies}

In this Section, we present the two missions under investigation and a formal description of the planning problems that appear. Both missions have objectives that need to be completed within a time window defined by the user which we call planning horizon. By designing and implementing a system based on 2 different missions, we aim at investigating whether it can be generalized to more Earth Observation missions.

\section{A. Disaster Monitoring Constellation 3 Earth Imaging Mission}

Surrey Satellite Technology Ltd (SSTL) Disaster Monitoring Constellation 3 (DMC3) is an Earth Imaging mission expected to be able to image daily a ground area of around 1 million square kilometers in the surface of the Earth. It consists of 3 spacecraft in a $650 \mathrm{~km}$ sun-synchronous orbit that are agile and can be steered up to $45^{\circ}$ off-nadir pointing along the roll and pitch axes. For this paper, we consider only roll axis steering. The angles take discrete values in the set $\left[-45^{\circ}, 45^{\circ}\right]$ starting from $-45^{\circ}$ and increasing by $5^{\circ}$.

There are 3 imaging operation modes but the most commonly used is the strip mode. In this mode, a "push broom" form of imaging is used i.e. the camera is lying across the orbit ground track, and the satellite captures long strips of orthogonal shape per flyby over an Area of Interest (AoI), as shown in Figure 1. The strip start and end times coincide with the time when a hypothetical line in the middle of a strip, that is parallel to the ground track of the spacecraft, enters and exits the AoI respectively. Hence, not all the strips of a flyby necessarily have the same start and end times e.g. when the roll angle $=0^{\circ}$, this line is the ground track. As a flyby time window we consider the time between the earliest start time and latest end time among the strips of the same flyby. Strips consist of smaller size images of square size 23 by 23 square kilometres, called scenes, which can be imaged within the roll capability range of the satellites. The width of a scene increases as the attitude of the spacecraft diverges from nadir, with a maximum width of about $44 \mathrm{~km}$.

Power and thermal constraints limit the maximum strip length to 175 scenes. The spacecraft have 2 data recorder devices: a small one of a total memory consisting of 30 GB meant to be used for near real time imaging, and one large device of a total memory of 512 GB meant to be used in a stored and forward manner 11 The size of a scene can vary depending on the attitude of the spacecraft, the location and compression used, from about 250MB to $1300 \mathrm{MB}$. The frequency of Ground Station (GS) passes is as high as one per day per Ground Station. Each imaged strip is initially stored in the on board memory. The satellites download data with a rate of $350 \mathrm{Mb} / \mathrm{s}$, thus their memory can be emptied after a pass with duration of at least 10 minutes. Ground Station passes duration can vary between 2-10 minutes.

One of the mission's objectives will be to image large areas, within the planning horizon. We define an area as large if multiple satellite flybys are needed to image it completely. A Coverage Planning problem consists of finding a way to cover all the parts of an area of arbitrary shape. In robotics, covering an area translates to visiting all of its points, thus a motion path has to be found for the robot. In Earth Imaging applications, a satellite is expected to image all the points of an area taking into account the available on board memory and the other existing constraints. When agile spacecraft are considered, a plan of the attitude maneuvers has to be decided. Regardless of the field of research, coverage planning is an $N P$-hard combinatorial optimization problem $\frac{12}{12}$

\section{Planning Problem definition}

In DMC3 mission, the users submit imaging requests to the MPS in the form of AoIs of arbitrary shape and size and the planning horizon. This information is combined with the DMC3 spacecraft orbits and times for the GS passes, the pointing availability of each spacecraft towards the AoI for the planning horizon given, and the initial available on board memory. The system outputs a chronological sequence of spacecraft 

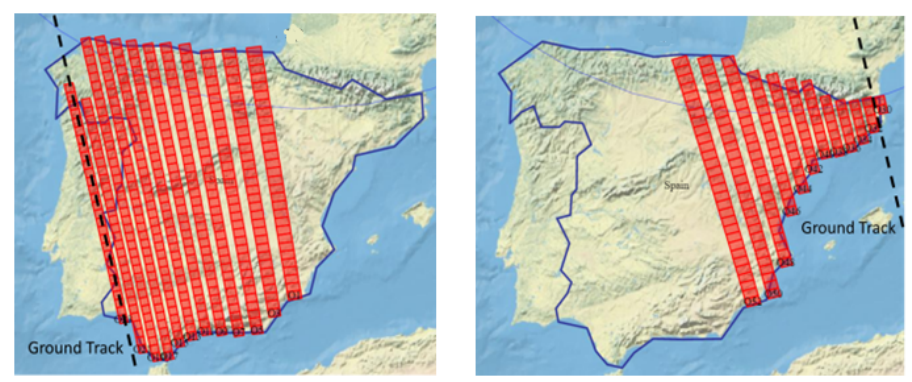

Figure 1. Two flybys over Spain with different pointing availability that is reflected to the number of available strips for each.

attitude at every flyby over the AoI. The objective that we investigate is the maximization of the percentage of the imaged area within the given planning horizon; the calculation of the imaged area's size is presented in 2 . The following constraints apply:

- Operational constraints. The fact that each spacecraft of the constellation has a given orbit is a constraint and determines the pointing availability of the spacecraft towards the AoI. The satellites are considered to maintain their attitude for the duration of the flyby over the AoI. Thus, the strips resulting from the possible tilt states are mutually exclusive with each other; at most one strip can be chosen for each flyby.

- Resource constraints. For the on-board storing, we only consider the use the small data recorder device; the images stored between GS passes must fit to it. Also, part of the resource constraints is defined by the GS' availability. GS are not guaranteed to be available during the time of a pass; their availability is subject to changes due to the workload or emergency cases. We only regard the GS passes as on board memory renewals, thus in case of availability change, the plan is adjusted. The resource constraints are satisfied by updating the current schedule once a newly added imaging opportunity results in memory overflow; this mechanism is described in detail in $\mathrm{B}$.

- Spatial constraints. Given that the ground track of the spacecraft is not the same at every flyby over an AoI and there are multiple spacecraft, it is certain that some acquired images will overlap with others, even for a single spacecraft. This will not only hinder or delay the total imaging of an area, but will also result in unnecessary use of the spacecraft imaging sensor. Nevertheless, overlap cannot be considered as a hard constraint of the problem, since a solution will still be feasible even when the images overlap. It is highly desirable to keep it at minimum levels, especially when the time horizon requested by the user for the completion of the task is not long.

- Transition time between two roll angles (slewing duration). When more than one target AoIs are considered, the slewing duration should be taken into account in the scheduling process. In specific, when two different target AoIs are adjacent to each other (with their latitude borders adjacent) one option is to regard the two AoIs as one and solve the problem. This poses the risk of poor imaging due to the strip length limitation - it is constrained to 175 scenes. On the other hand, regarding them as two different AoIs requires consideration of the transition time, as for some flybys the chosen roll angles for the two AoIs can be different. The transition time between the roll angles implies less time for imaging. In this paper, we consider that the target AoIs are not adjacent, and that there is time for switching from one roll angle to another without affecting the time for imaging.

\section{Coverage and Overlap calculation}

Computing the size of the imaged area for a given combination of strips can become a bottleneck for the performance of the system. The underlying geometric problem involves the calculation of the size of each strips intersection with the AoI, subtracting the overlapping areas i.e. areas covered by two or more strips. This calculation is too computationally expensive to be used many times. We estimate the coverage employing a simple algorithm that is based in one assumption: given that DMC3 is imaging only in ascending node, for an AoI of sufficiently small size, the Earth's curvature is small enough to assume that the ground 
tracks of the spacecraft are not affected by it and are almost parallel to one another. Thus, it is safe to assume that the orientation of the all the strips in the search space will be roughly the same, and any overlap area among the strips will be orthogonal.

The calculation involves dividing the AoI in bands parallel to each other, with a band frame orientation equal to the average orientation of the ground tracks (of all the flybys within the planning horizon) with respect to a Cartesian frame. For sufficiently narrow bands, if two strips intersect with the same band, they are expected to overlap with each other. The width of the bands is equal to $1 / 5$ of the narrowest strip of the problem.

For each strip combination though, we only consider the width that it can cover, based on the maximum and minimum longitude coordinates of the combination. We maintain and update two tables, each with size $\mathrm{B}$, equal to the number of bands considered. In the two tables we keep the maximum and minimum latitude values of all the strips intersecting each band, and the second biggest and second smallest latitude values correspondingly. To find the bands that each strip intersects with, we use binary search for its leftest longitude coordinate with respect to the positions of the bands. The sum of the differences of maximum and minimum latitude coordinates for all bands is an estimation of the area that is imaged by one or more strips. On the other hand, the sum of the differences of the second maximum and minimum latitude coordinates for all bands is an estimation of the area that is imaged by more than one strips. Thus, the difference of these two values is the size of the area imaged by a single strip. We aim at maximizing this quantity, the area imaged by a single strip.

\section{B. Data Relay Mission}

The increase in the EO missions population has not only led to producing automated MPS, but also inevitably results in a corresponding increase of the amount of data produced on board; this data needs to be transmitted to the ground as soon as possible in order to free on board resources, or satisfy an urgent user request. To that purpose, Data Relay missions are being designed and operated. In those missions, GEO spacecraft act as relays of data among LEO spacecraft and Ground Stations (GS); LEO data are sent to a GEO spacecraft and then transmitted to a Ground Station. As a result, communication among LEO and GS takes place with reduced time delays in the transmission, almost regardless of their relative position. NASA's Tracking Data Relay Satellites System (TDRSS) ${ }^{13}$ and ESA's European Data Relay System (EDRS) 14 are two examples of such missions.

\section{Planning Problem definition}

A Data Relay mission planning system determines whether and when each LEO spacecraft will be given communication time on the GEO satellite; this is an oversubscribed scheduling problem. In this family of problems, the initial set of tasks e.g. communication tasks, is larger than the set of the tasks that can be scheduled, regardless of the planning horizon. A simplified model of the problem is considered, in which 1 GEO spacecraft acts as a relay, and $N$ LEO spacecraft users submit requests for communication, called links. Only the link requests that arrive prior to the start of the planning process are considered. Each link is associated with the following values:

- Earliest start time, e.s.t

- Latest end time, l.e.t

- Duration, dur

- Priority, $p$

We call the time window l.e.t. - e.s.t. a feasibility window. All values are positive and fixed for each link

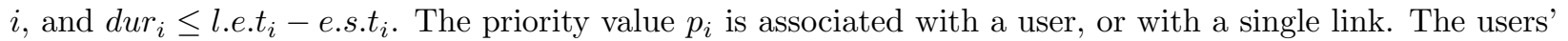
requests are expected to be scheduled within the planning horizon, a typical duration for which is 1 day. The problem constraints are:

1. For every link, its e.s.t. and l.e.t. cannot be smaller or larger than the lower bound and upper bound of the planning horizon respectively. 
2. No link can start before the earliest start time, or finish after the latest end time. If $t_{s}$ is the actual start time of a link, then e.s.t $\leq t_{s} \leq$ l.e.t - dur.

3. The link requests cannot be split into several parts.

4. A gap of $k$ time units between two consecutive scheduled links must be considered.

5. Two links cannot be accommodated at the same time. The GEO resource is binary, thus for the actual start time $t_{s}$ of all $i, j$ links where $i \neq j$, and taking into account constraint 3 , we have either $t_{s i}+d u r_{i}+k<t_{s j}$ or $t_{s j}+d u r_{j}+k<t_{s i}$.

The individual requests are feasible i.e. both the LEO spacecraft and the GS they aim at transmitting their data to, are within the field of view of the GEO satellite during the whole feasibility window. The problem objective is to maximize the sum of the scheduled links' priorities. The capability of the mission is the setting up of the communication link between a specific LEO spacecraft and the requested GS. Thus, the system output consists of a sequence of commands that dictate the order in which specific communication links will be set up.

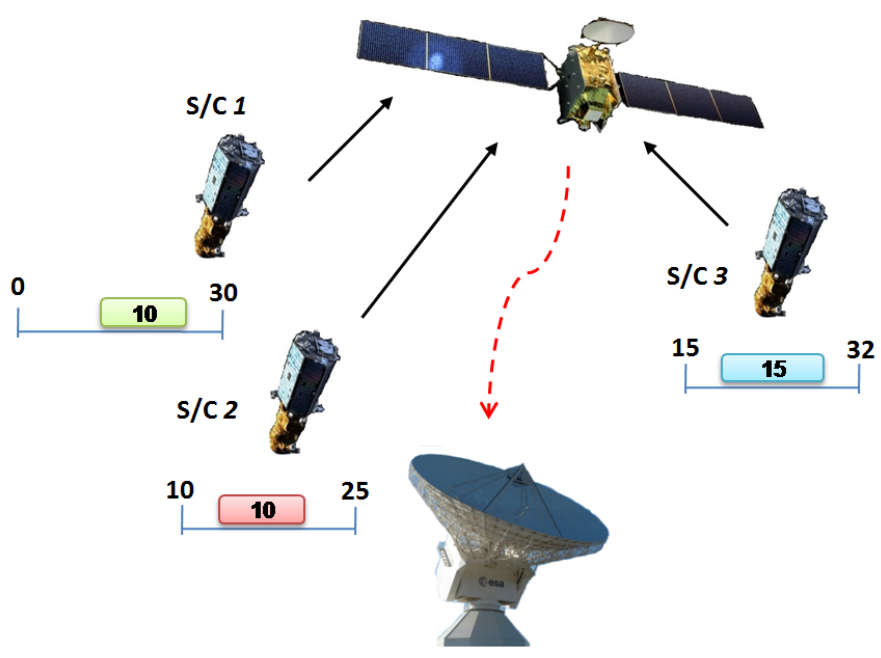

Figure 2. Data Relay mission configuration.

An example of a Data Relay mission configuration with 3 LEO spacecraft is shown in Figure2 2 The link request from Spacecraft 1 has e.s.t. $=0$,l.e.t. $=30$ and $d u r=10$. The corresponding values for Spacecraft 2 and 3 are e.s.t.2 $=10$,l.e.t. ${ }_{2}=25$, dur $r_{2}=10$ and e.s.t. $3=15$,l.e.t. $3=32$, dur $r_{3}=15$.

\section{Mission Planning System Architecture}

In this Section, we describe the Mission Planning System architecture and requirements, the problem representation and the optimization algorithm. The description is kept as abstract as possible and mission specific details are mentioned when needed. Fig.3 shows the high level MPS architecture. The users submit their requests to a certain mission, defining all the mission specific details. The requests are combined with the Mission's Capabilities, Constraints and Objectives in a problem model. The model is then inserted to the $\mathrm{P} \& \mathrm{~S}$ algorithm and a solution is produced.

In accordance to Fig. 3, on Table 1 we define the System Input, and Output for the planning problems described.

\section{A. Problem Representation}

In order to apply an Ant Colony Optimization algorithm to an optimization problem, the problem environment must be translated to a graph. Thus, the Input information is modelled using a directed graph 
Mission Input

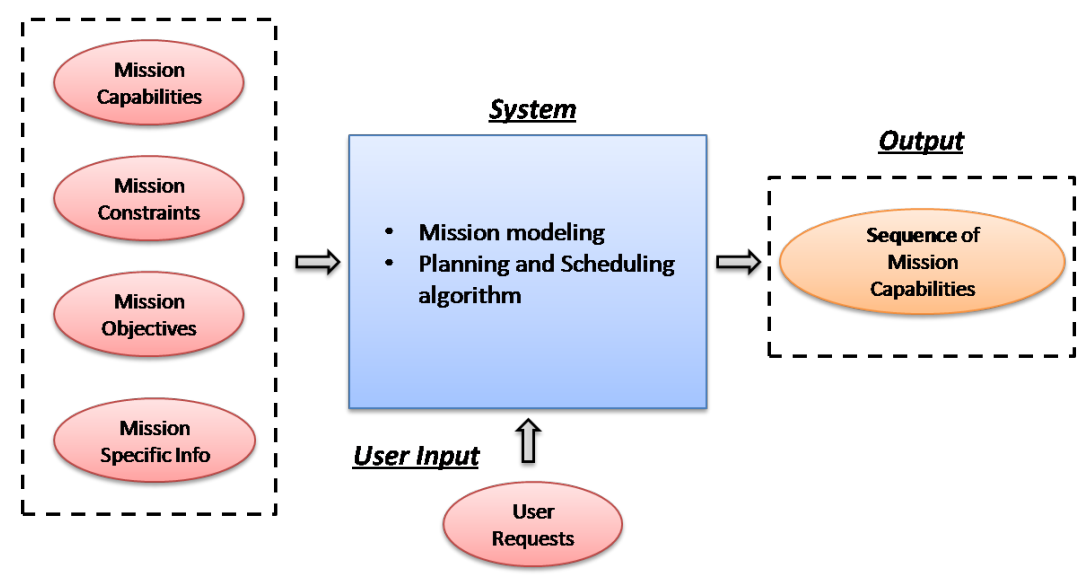

Figure 3. Autonomous Mission Planning System

\begin{tabular}{c||c|c} 
Input Information & Coverage Planning & Data Relay \\
\hline \hline Mission Capabilities & $\begin{array}{c}\text { Up to } 45^{\circ} \text { steering off-nadir } \\
\text { pointing on the roll axis }\end{array}$ & $\begin{array}{c}\text { Setting up communication links } \\
\text { between LEO s/c and GS }\end{array}$ \\
\hline Mission Constraints & $\begin{array}{c}\text { a) Binary resource (1 imaging sensor), } \\
\text { Resource Capacity: Limited on-board memory } \\
\text { b) S/C Orbits and GS passes' times } \\
\text { c) Same attitude per flyby } \\
\text { d) Image Overlap }\end{array}$ & a) Binary Resource \\
& $\begin{array}{c}\text { Maximize the Imaged Area } \\
\text { Mission Objectives }\end{array}$ & $\begin{array}{c}\text { Maximize the sum of the } \\
\text { scheduled links' priorities }\end{array}$ \\
\hline Output & A chronological sequence of \\
images (scenes) & $\begin{array}{c}\text { A chronological sequence of } \\
\text { links to be set up }\end{array}$
\end{tabular}

Table 1. System Input Information and Output

representation, which constitutes the input to the optimization algorithm. A directed graph $\mathcal{G}=(\mathcal{V}, \mathcal{E})$ consists of:

- A set of nodes, $\mathcal{V}$. Each node can be associated with:

1. an id

2. the set of the incoming edges

3. the set of the outgoing edges

4. a weight

5. additional information

- A set of directed edges, $\mathcal{E}$, connecting nodes. The edges are directed from one node to another. A pair of nodes can be connected by more than one edge. Each edge can be associated with:

1. an id

2. the id of the origin node

3. the id of the destination node

4. a weight 
5. additional information

The optimization algorithm traverses the graph and produces a solution or a path. A path is a set of edges with the same direction, connecting a sequence of nodes of the graph. It is worth noting that not all the nodes must be included in the path, that depends on the objective of the search. In Table 2 we describe the graph construction for the two different problems. The objective function depends on the nodes and/or the edges of the solution path.

\begin{tabular}{c||c|c}
\multicolumn{1}{c||}{ Graph Component } & Coverage Planning & Data Relay \\
\hline \hline Node & Flyby over AoI / GS pass & Link \\
\hline Node Weight & $\begin{array}{c}\text { Capacity of memory renewal } \\
\text { (for GS passes) }\end{array}$ & - \\
\hline Edge Add. Info & Type (Flyby / GS pass) & $\begin{array}{c}\text { Feasible combination of } \\
\text { origin and destination Nodes }\end{array}$ \\
\hline Edge Weight & $\begin{array}{c}\text { Objl angle of imager (Strip) } \\
\text { for the object the edge represents }\end{array}$ & $\begin{array}{c}\text { Objective function evaluation } \\
\text { for the Node the edge is directed to }\end{array}$ \\
\hline Edge Add. Info & Strip Memory consumption & $\begin{array}{c}\text { Value for gap required before } \\
\text { and after the link }\end{array}$ \\
\hline Start Node & The chronologically first Flyby or GS pass & Discussed in C \\
\hline End Node & The chronologically last Flyby or GS pass & Not specified \\
\hline "No" edge & Yes & No \\
\hline
\end{tabular}

Table 2. Graph Components

The graph for the Coverage Planning problem consists of $N$ nodes, where $N$ is the number of flybys over the AoI within the planning horizon. The nodes are ordered chronologically from earliest to latest flyby times. Each node has a set of incoming edges to it, each corresponding to a roll angle choice for this flyby. For all the nodes there is an extra edge representing the option of No imaging during the flyby. Thus, the memory constraint does not allow for all the flybys to image the AoI, the ants choose the 'No' edge. In between the nodes representing the flybys, we add nodes that represent the GS passes, in the correct chronological order. Figure 4 shows an example for 3 flybys and 1 GS pass.

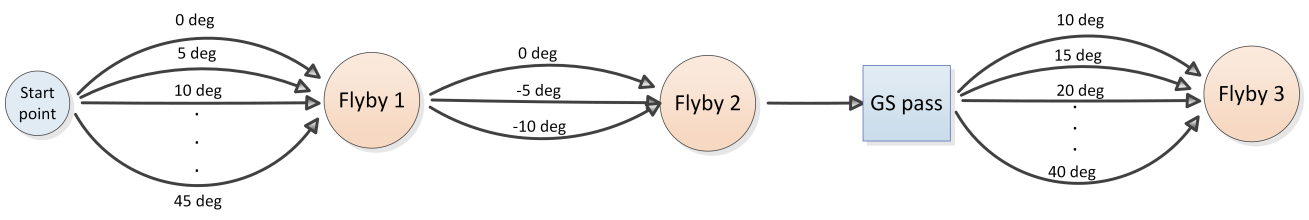

Figure 4. Example of graph representation for the Coverage Planning problem

In the Data Relay case, the graph has $M$ nodes, where $M$ is the number of submitted link requests. An edge from node $i$ to node $j$ is added if

- e.s.t. ${ } \geq$ l.e.t. ${ }_{i}$, or

- e.s.t. $i+$ dur $_{i}-1+k+$ dur $_{j}-1 \leq$ l.e.t.j

Correspondingly, an edge from node $j$ to node $i$ is added if

- e.s.t. ${ }^{2} \geq$ l.e.t.j, or

- e.s.t.j + dur $_{j}-1+k+$ dur $_{i}-1 \leq$ l.e.t. $i$

Simply, if an edge is incoming to node $i$, link $i$ can succeed link $j$ in a schedule, and vice versa. Essentially, in this graph representation each pair of connected nodes forms a feasible combination of links. In Figure 5 we present the graph for the example shown in 2 . 


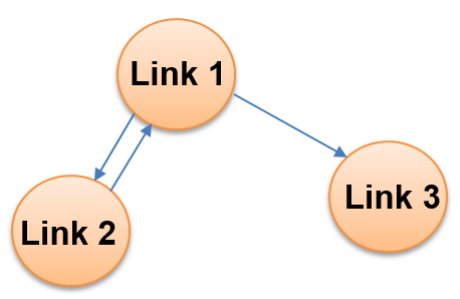

Figure 5. Graph representation for the Data Relay System configuration shown in 2

\section{B. Planning with Ant Colony Optimization}

Real world ant colonies find the shortest paths between their nests and a food source communicating indirectly via the environment. The ants first wander randomly searching for food but upon finding it, they return to their colony laying down pheromone in the path they followed. When other ants sense this trail, they are likely to follow the whole or part of the path, reinforcing it with pheromone too if it leads them to food. The trails evaporate with time, thus the total amount of pheromone in shorter paths is higher, since they get marched over more frequently. Even though the individuals follow simple sets of rules and do not have universal knowledge of the colonys actions, the colony presents a complex behaviour, solving the ants' foraging problem.

This mechanism is used in optimization problems that can be reduced to finding optimal paths in graphs ${ }^{15}$ The meta-heuristic method is called Ant Colony Optimization (ACO) and is very often met in Computer Science and Operations' optimization problems. A typical ACO algorithm involves three main steps:

1. Path Construction: The ants construct a path starting from an initial node and iteratively adding an edge to it, choosing among their options. The probabilistic rule they use favours the edge with the highest pheromone amount:

$$
P_{i j}=\frac{\tau_{i, j}^{\alpha} * \eta_{i, j}^{\beta}}{\sum_{j=1}^{M_{i}} \tau_{i, j}^{\alpha} * \eta_{i, j}^{\beta}}
$$

where $\tau_{i, j}$ is the amount of pheromone in the edge connecting nodes $i$ and $j$ (or edge $i j$ ), the heuristic $\eta_{i, j}$ is the value weight of the same edge and $M_{i}$ is the number of incoming edges in the node $i$. Parameters $\alpha$ and $\beta$ define the importance of the pheromone and the heuristic, respectively, in the ants' choices. (1) is implemented in the system as a roulette wheel selection process; the slice of each edge in the wheel is proportional to the value of (1) for this edge and the a slice is chosen randomly with a random number generator.

2. Path Evaluation: When a path is constructed, each ant evaluates it based on an objective function, $f$.

3. Update of pheromone field: The update is twofold: each ant deposits an amount of pheromone to the path it constructed, based on its evaluation. The better the path evaluation, the more the pheromone deposited. Then, the pheromone on all the edges of the graph is evaporated by a fixed rate, $\rho=0.05$.

In the ACO algorithm we employ, a mechanism to control the balance of exploration and exploitation phases is used. During the exploration phase the goal is to analyse unexplored areas of the search space, whereas during the exploitation phase the search focuses on regions that include the best solutions found to time, so as to fine tune them by searching close to them. Algorithm 1 shows the high level workflow.

In the pherFieldInit function we assign all edges with the same amount of pheromone in the beginning of the run. The startNode() function defines the node from where the search will begin. As shown in Table 2. in the Coverage planning problem the start node is the one that represents the chronologically first flyby over the AoI. For the Data Relay planning problem though, the first link of the schedule can start close to the lower bound of the planning horizon, since the objective is a non decreasing function of the time the GEO resource is in use. Thus, having a solution with the first link starting e.g. in the middle of the planning horizon can be a risk to finding good solutions. We choose the first link among the ones that have an e.s.t. $<l$ using a weighted roulette that favours the link that optimizes the objective function. The value

$$
8 \text { of } 11
$$

American Institute of Aeronautics and Astronautics 


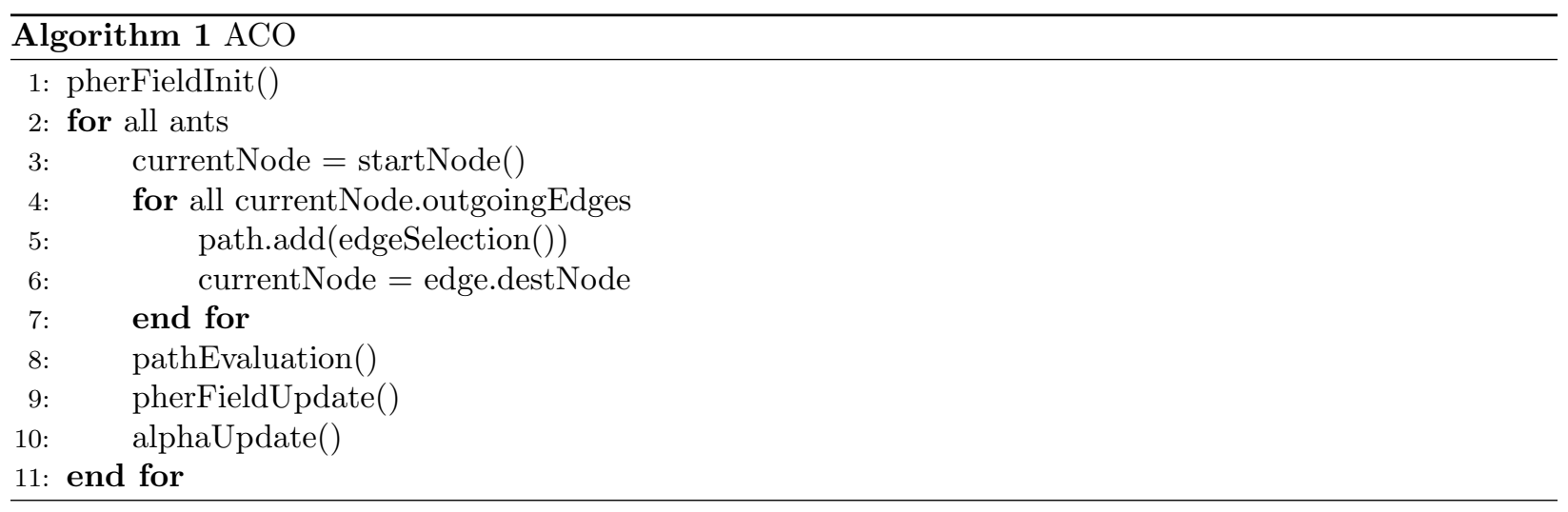

of $l=20$ is empirically found to bring better results.

Out of all the outgoing edges of this node, we select one and proceed to the next node with edgeSelection(). The selection is based on the probabilistic rule (1). In this function, the constraints (a) and (b) from Table 1 for Coverage Planning and Data Relay respectively are taken into account. In the Coverage Planning problem, every time a newly added edge - that translates to a newly added strip in the schedule - results in memory overflow, we use a weighted roulette mechanism to choose which of the edges of the current path will be deleted. Only the part of the path since the last GS pass is considered, since at the GS pass the on board memory, or part of it, was renewed. After the deletion, we repeat the process in case the constraint is still violated. The path construction stops once the end node of the graph is visited. In the Data Relay System case, the overlap constraint is partly integrated in the graph, with each pair of connected nodes representing a feasible combination of links. When choosing a node, we fix its start time to the earliest possible available time e.g. the start node will be scheduled on its e.s.t. Among all the outgoing edges of a node, only the ones that lead to links that can be added to the schedule are considered for selection. For example, if a schedule consists of links $\{4,6,2,9\}$ (link IDs) in a chronological order, and link 9 has an edge directed to link 1, then if $\{4,6,2,9,1\}$ is not a feasible combination of links - even though $\{9,1\}$ is a feasible combination - then this edge will not be considered in the weighted roulette mechanism for selection. In case the roulette is empty, the path construction is completed. Otherwise, the path construction continues by adding the gap of $k$ minutes at the end of the current schedule, and then choosing the next node that will be added.

The created path is evaluated by an objective function, $f$, based on which we update the pheromone field, by evaporating and depositing an amount of pheromone. In the alphaUpdate() function, the value of alpha increases in order to increase the pheromone field effect in the ants' choice, based on (1). This is an online adjustment of the exploration/exploitation balance found $\frac{16}{16}$ to lead the system to convergence.

\section{Differences in the planning process between the two different missions}

In previous work 17,18 we have applied the ACO algorithm described in B to the two planning problems, providing some preliminary results on the system's performance. In this paper, apart from proposing a novel graph representation for the Data Relay planning problem (described in A), we compare and contrast the planning process for the two Case Studies. The differences are found in the:

- Constraint satisfaction: In both cases, the paths produced are feasible, thus the constraints are satisfied during the solution construction, in the edgeSelection function. The difference lies in the time we deal with the resource constraints (Constraints (a) on Table 1). In Coverage Planning, the fact that the resource is binary is dealt with by having flyby windows that do not overlap. Regarding the resource capacity constraint though, we only act in the event that it is violated i.e. if the plan constructed until that moment would result in memory overflow if executed. In the Data Relay problem, on the other hand, the binary resource constraint is satisfied by decreasing the options of edges that are eligible for selection (described in $B$ ) and is, thus, never violated.

- Start node choice. The start Node is fixed in the Coverage Planning problem or different for each ant in the Data Relay case.

- "No" edge presence. In the Coverage Planning problem representation, each ants traverses the

$$
9 \text { of } 11
$$


whole graph, but it is not mandatory for the mission to take an image at every flyby. Thus, for each pair of nodes there is an additional edge that represents the option of not imaging. On the other hand, in the Data Relay case, each ant can search different parts of the graph, and most probably not all of it.

The rest of the planning process has no difference for the two Case Studies presented.

\section{Conclusions and Future Work}

In this paper we propose a Mission Planning System architecture that is based on the ants' foraging mechanism to address two different planning problems. We investigate the similarities and differences in the planning process for each problem: the first is a Coverage Planning problem from an Earth Imaging mission, DMC3 from SSTL, and the second is a typical Oversubscribed Scheduling problem for unit capacity resources that can be found in Data Relay missions. There are various constraints and objectives that a space mission can have, thus it is difficult to strictly determine the types of planning problems the proposed MPS can address. We generalise on that by referring to the two missions described in this paper and constraints also discussed on. 10 In both cases presented in this paper, the constraints are satisfied during the construction of the candidate solution paths. Those are:

- Capacity Constraints. The resources considered in the problem are binary, thus they are either occupied or not and a task cannot overlap another that is occupying this resource. In the Coverage Planning problem, there is another capacity resource constraint, the limited on board memory of the spacecraft. The more tasks are added to the schedule, the less the available memory. When downlink activities take place, the memory is renewed. The mechanism for satisfying this constraint can be found in Section B.

- Temporal Constraints:

1. Tasks that have earliest start and latest end times and fixed durations.

2. Relative time or predefined ordering of any two tasks. In the Coverage Planning problem the ordering of the tasks coincides with their chronological order and is integrated in the problem representation, with a chain-like graph. Information regarding relative time distance between two tasks, e.g. task $B$ must take place at least 10 minutes after task $A$, can be included in the edges in the way the gap between two links is currently included in the Data Relay case, described in B.

- Mutually exclusive tasks. This constraint is integrated in the graph, with mutually exclusive tasks not allowed to appear in the same paths.

Thus, the MPS system presented can solve problems that can be reduced to graph search problems that have the aforementioned constraints. There is no restriction regarding the problem objectives or the heuristic used, which can be equal to the objective function evaluation for a single edge, like in the two problems of the paper.

Future work for this project sees the application of the ACO approach proposed in this paper, to the coordination of multiple spacecraft in a constellation mission. The additional constraint is that the same task cannot be accommodated by more than one spacecraft. The coordination mechanism should allow for the system to adapt to any type of requests, not allowing for duplicate links or extreme load imbalance among the GEO resources and should also be scalable to both the number of requests and resources. Thus, we adjust the current MPS by having multiple graphs, one for each spacecraft, which are considered as a unit due to the paths' evaluation by an objective function that is common for all.

\section{Acknowledgments}

This work is co-funded by the Surrey Space Centre (SSC) of the University of Surrey, the Surrey Satellite Technology Ltd (SSTL) and the Operations Centre of the European Space Agency (ESA/ESOC). 


\section{References}

${ }^{1}$ Smith, B., Engelhardt, B., and Mutz, D., "The RADARSAT-MAMM Automated Mission Planner," AI Magazine, Vol. 23(2), 2002, pp. 2536.

${ }^{2}$ Maurer, E., Mrowka, F., Braun, A., Geyer, M., Lenzen, C., Wasser, Y., and Wickler, M., "TerraSAR-X Mission Planning System: Automated Command Generation for Spacecraft Operations," IEEE Transactions on Geoscience and Remote Sensing, Vol. 48(2), 2010, pp. 642648.

${ }^{3}$ Policella, N., Oliveira, H., and Siili, T., "Managing SOHO's Keyhole Periods: Problem Definition and Solving Model," Proceedings of the International Workshop on Planning and Scheduling in Space, Pasadena, California, USA, 2009.

${ }^{4}$ Johnston, M. D. and Giuliano, M., "Multi-Objective Scheduling for the Cluster II Constellation," 6th International Workshop on Planning and Scheduling in Space, 2011.

${ }^{5}$ Iacopino, C., Palmer, P., Brewer, A., Policella, N., and Donati, A., "EO Constellation MPS Based on ant Colony Optimization Algorithms," 6th International Conference on Recent Advances in Space Technologies - RAST2013, IEEE Press, Piscataway, NJ, 2013, pp. 159-164.

${ }^{6}$ Dvorak, D. D., Ingham, M. D., Morris, J. R., and Gersh, J., "Goal-Based Operations: An Overview," Journal of Aerospace Computing, Information, and Communication, Vol. 6(3), 2009, pp. 123-141.

${ }^{7}$ Uhlig, T., Sellmaier, F., and Schmidhuber, M., Spacecraft Operations, Springer, 2015.

${ }^{8}$ Tejo, J., Pereda, M., Veiga, I., Chamoun, J., Garcia, G., and Beech, T., "flexplan: An Operational Mission Planning \& Scheduling COTS Used Internationally," 5th International Workshop on Planning and Scheduling in Space, Baltimore (USA), 2006.

${ }^{9}$ Donati, A., Policella, N., Cesta, A., Fratini, S., Oddi, A., Cortellessa, G., Pecora, F., Schulster, J., Rabenau, E., Niezette, M., and Steel, R., "Science Operations Pre-Planning \& Optimization using AI constraint-resolution - the APSI Case Study 1," SpaceOps 2008, 2008.

${ }^{10}$ Chien, S. A., Johnston, M., Frank, J., Giuliano, M., Kavelaars, A., Lenzen, C., and Policella, N., "A generalized timeline representation, services, and interface for automating space mission operations," SpaceOps 2012, Stockholm, Sweeden, 2012.

${ }^{11}$ Iacopino, C., Harrison, S., and Brewer, A., "Current and Future Challenges in Mission Planning Systems for Commercial Earth Observation Constellations," International Workshop on Planning and Scheduling for Space (IWPSS 2017), Pittsburgh, PA, jun 2017.

${ }^{12}$ Strimel, G. P. and Veloso, M. M., "Coverage Planning with Finite Resources," International Conference on Intelligent Robots and Systems(IROS), 2014.

${ }^{13}$ Gramling, J. and Chrissotimos, N., "Three Generations of NASA's Tracking and Data Relay Satellite System," SpaceOps 2008, Heidelberg, Germany, 2008.

${ }^{14}$ Wallrapp, F., Ballweg, R., and Gataullin, Y., "The European Data Relay System (EDRS): Operational Challenges," 62nd International Astronautical Congress, Cape Town, SA, 2011.

${ }^{15}$ Dorigo, M. and Stutzle, T., Ant Colony Optimization, MIT Press, 2004.

${ }^{16}$ Iacopino, C. and Palmer, P., "The Dynamics of Ant Colony Optimization Algorithms Applied to Binary Chains," Swarm Intelligence, Vol. 10, 2012, pp. 343-377.

${ }^{17}$ Ntagiou, E., Armellin, R., Iacopino, C., Policella, N., and Donati, A., "Coverage Planning for Earth Observation Constellations," International Conference on Automated Planning and Scheduling - Scheduling and Planning Applications woRKshop, Pittsburgh, PA, 2017.

${ }^{18}$ Ntagiou, E., Armellin, R., Iacopino, C., Policella, N., and Donati, A., "Ant Colony Optimization applied to the Planning of a Data Relay Space Mission," International Workshop on Planning and Scheduling in Space, Pittsburgh, PA, 2017. 